\title{
A rare involvement in Behcet's Disease: Carotid artery aneurysm
}

\author{
Hamit Serdar Basbug, Yalcin Gunerhan, Hakan Gocer, Kanat Ozisik \\ Department of Cardiovascular Surgery, Kafkas University Faculty of Medicine, Kars, Turkey
}

A 20-year-old male was admitted to the outpatient clinic with the complaint of a mass on the left side of his neck. According to the anamnesis, the mass had initially appeared 1 year earlier and slowly grew larger. He had a 4-year history of relapsing oral and genital ulcers that was diagnosed as Behcet's disease 2 years prior. He had impaired vision in his left eye and a minimal sense of light in his right eye. Physical examination revealed a pulsatile mass on the left side of his neck (Figure 1). Color Doppler ultrasound examination revealed a true aneurysm of the left carotid artery with dimensions of $27.5 \mathrm{~mm} \times 20.3 \mathrm{~mm}$ (Figure 2). Transverse slice of computerized tomography (CT) angiography demonstrated an aneurysm at the level of the left carotid bifurcation (Figure 3). A 3-dimensional reconstruction of the CT angiography views illustrated a saccular aneurysm and its relationship to the skeletal system (Figure 4A) and to vascular structures only (Figure 4B), indicating that the aneurysm was discrete and did not involve the aortic arch or other great vessels. The patient was prescribed prednisone (80 mg/day), azathioprine (150 mg/day), colchicine $(1 \mathrm{mg} /$ day), and acetylsalicylic acid (150 mg/ day), and discharged upon his rejection of surgical or endovascular treatment.

Behcet's disease is a systemic immune vasculitis that often presents with recurrent oral and genital mucous membrane ulcers (aphthae) accompanied

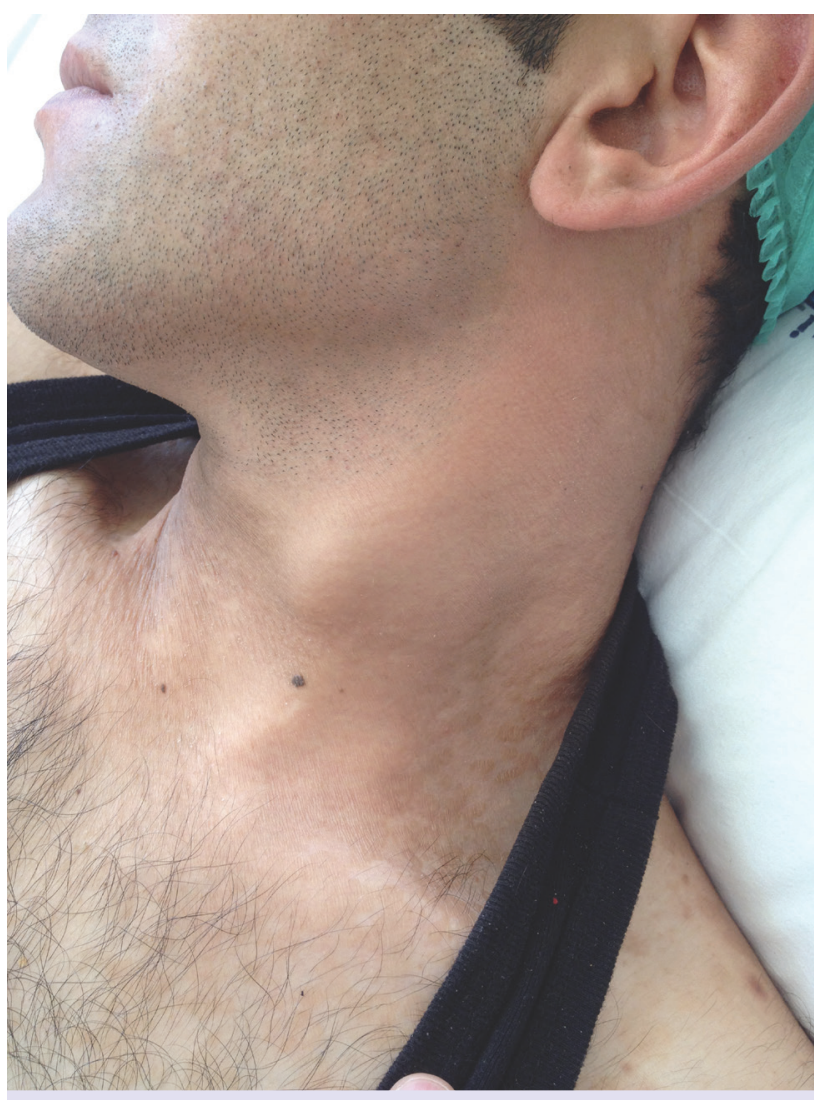

FIGURE 1. Morphological view.

by skin lesions, gastrointestinal involvement, ocular disease, neurological manifestations, vascular pathologies, or arthritis. Although some compo-

Received: January 23, 2017 Accepted: March 23, 2017 Online: August 26, 2017

Correspondence: Dr. Hamit Serdar BASBUG. Kafkas Universitesi Tip Fakultesi Kalp ve Damar Cerrahisi Pasacayiri, 36100 Kars, Turkey. 

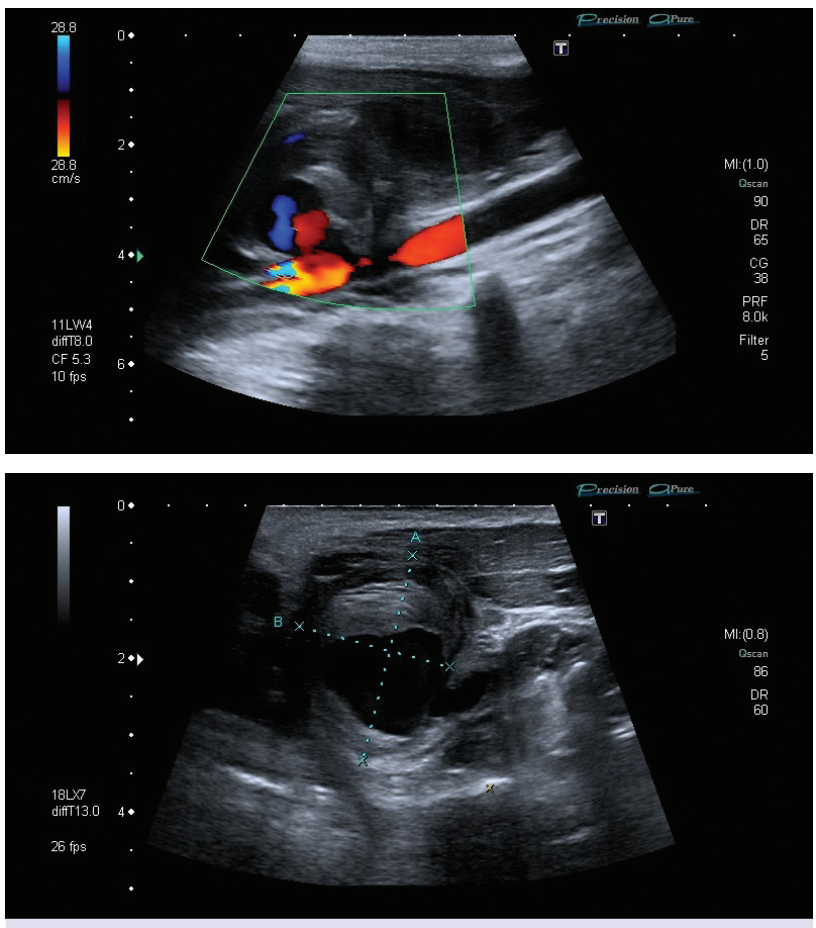

FIGURE 2. Color Doppler ultrasound view showing the true carotid aneurysm (top) and the diameter (bottom).

nents of Behcet's may have been referenced by Hippocrates, the disease was first fully described and brought to the attention of medical society by Hulusi Behcet in 1937. Clinical manifestations of Behcet's disease are mostly due to systemic vasculitis. Compared to all other systemic vasculitides, Behcet's disease is notable for its potential to affect blood vessels of all sizes (large, medium, and small) on both the venous and arterial side of the vasculature. Deep venous thrombosis and thrombophlebitis are the most frequent vascular manifestations, followed by an arterial aneurysm and obstruction. Arterial aneurysms mostly occur in the abdominal aorta and pulmonary arteries. According to the recent literature, less than 50 cases of extracranial carotid artery aneurysm have been reported. In conclusion, vascular complications are the most important predictors of mortality and morbidity in Behcet's disease. Appropriate medical treatment should be constituted as far as possible and surgical and endovascular treatment options should be evaluated without delay before a possibly irreversible complication occurs.

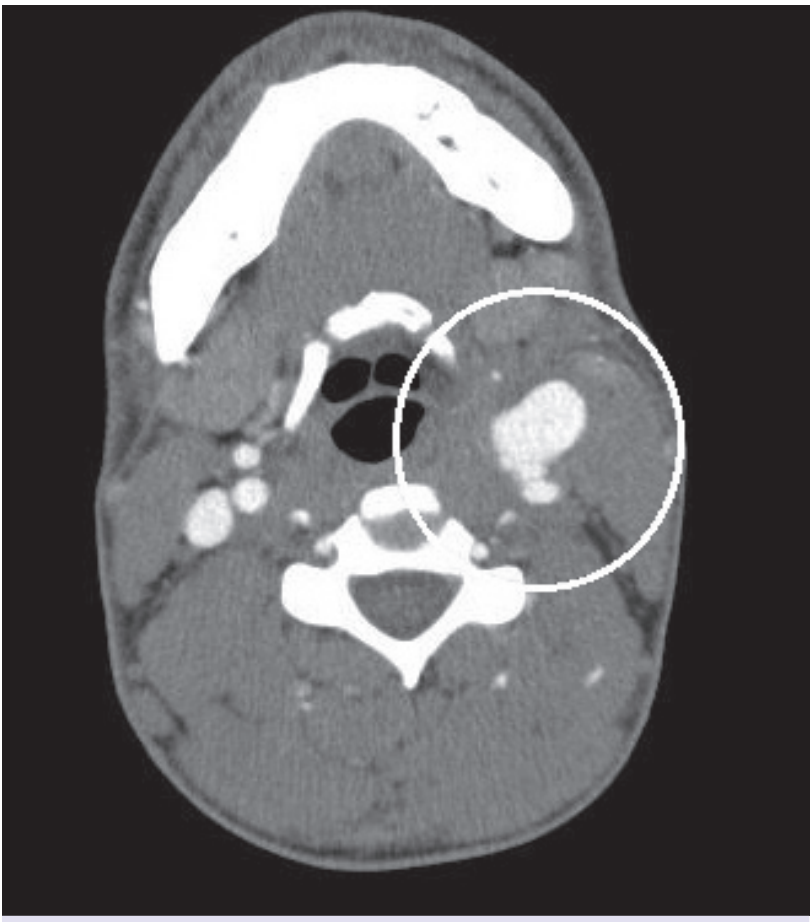

FIGURE 3. Computed tomography angiography transverse slice revealing the carotid aneurysm.
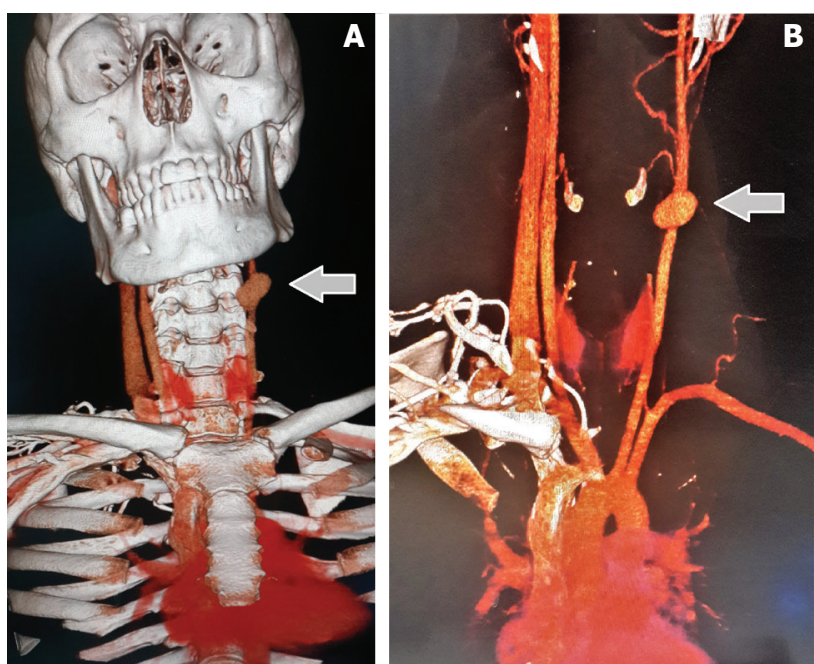

FIGURE 4. (A) Computed tomography (CT) angiography (3-dimensional reconstruction with vascular and bony structures) illustrating the relationship of the carotid aneurysm to the skeletal system. (B) CT angiography (3-dimensional reconstruction with only vascular structures) indicating that the carotid aneurysm was discrete and that there was no accompanying aneurysm(s) of the great vessels. 